\title{
An Introduction of the Role of Virtual Technologies and Digital Twin in Industry 4.0
}

\author{
Mohammad Azarian, Hao Yu, Wei Deng Solvang and Beibei Shu ${ }^{1}$
}

\begin{abstract}
It is inevitable that technological improvements have affected human's life in any aspect to a large extent. Automation, artificial intelligence, robotics, etc., are some advances contributed to the fourth industrial revolution: Industry 4.0. Despite there are still many arguments, Internet of Things (IoT) and Cyber Physical Systems (CPS) have been widely acknowledged as the main fundaments of Industry 4.0. This paper introduces the concept of CPS by providing an explicit framework that unifies the existing theories in this regard. Nine exquisite technologies attributed to Industry 4.0 are investigated, among which virtual technology (VT) and digital twin (DT) are considered as two of the core criteria and are thus focused on in this paper. However, for providing an acceptable level of integration and intelligence, noticeable gap between the virtual world and the real factory is still a significant challenge. Holistic approaches addressing this issue suggest VTs and DT have the potential to form the fundaments for more improvements in terms of both interoperability and consciousness. Furthermore, they may pave the way for achieving the highest level of CPS and Industry 4.0.
\end{abstract}

Keywords: Industry 4.0, Cyber Physical System, Virtual Technology, Digital Twin

\section{Introduction}

The history of manufacturing industry has shown that the emergence and application of new technologies is the most important driving force in determining the turning point and structural alterations. The first industrial revolution occurred thanks to the invention of steam engine; the use of electricity drove the second industrial revolution by enabling mass and standardized production. The combination of IT technologies and electronic devices contributed to the automation in the third industrial revolution [1] [2]. The increasing rate of customization and demand diversity has led factories and manufacturing companies to become more specialized and smaller in many regions [3] [4]. In order to survive in today's competitive global market, manufacturers have to

\footnotetext{
${ }^{1}$ Mohammad Azarian ( $\square$ ), Hao Yu, Wei Deng Solvang and Beibei Shu Department of Industrial Technology, Faculty of Engineering Science and Technology, UiT Arctic University of Norway, Narvik, Norway azarian135@gmail.com \{hao.yu, wei.d.solvang, beb ei.shu\}@uit.no
} 
focus on the advances of both technologies and manufacturing theories, which further leads to the shift to a novel manufacturing paradigm: Industry 4.0.

Since the introduction of Industry 4.0 (I4.0 in this paper) at Hannover fair in 2013, different manufacturing ideas and technologies have been developed, among which Intelligent Analytics and Cyber Physical Systems (CPS) are unified as two major technical drivers of I4.0 [5]. An evaluation on CPS dimensions indicates that it internally encompasses most of the existing technologies, which are believed as the nine fundamental elements of I4.0 [6]. Furthermore, research works related to those nine elements suggest that Internet of Things (IoT) not only is committed to provide a widespread connectivity but also forms the basis for boosting the overall intelligence and integration level [7] [8] [9]. As a result, CPS and IoT can be considered as two major fundaments to form the technological structure of I4.0.

The elements of the CPS mainly contribute to increase the interoperability and consciousness of a manufacturing system. More precisely, they focus on incorporating all machines and components in a cyber environment and then add consciousness to the system in order to increase the intelligence level within the unified factory [10]. In this regards, this paper investigates the interoperability aspect and evaluates the confronting challenges. Section 2 presents a structural framework for clearly demonstrating the steps to be taken in order to achieve I4.0. Through providing a case study, section 3 discusses the role of virtual technologies and simulation. In order to tackle the most significant challenge, section 4 puts forward the concept of digital twin so that a bidirectional connection between the virtual world and the physical factory can be established. Section 5 concludes the paper.

\section{Cyber Physical System}

CPS consists of physical components and machines, which are interconnected. The associated data is collected in an integrated cyber environment, which brings the opportunity for remote control and data processing [11] [12]. Many believes that the implementation of CPS is the key element to achieve I4.0. In a comparison between the current status of manufacturing and the future production system in I4.0, component, machine and production systems are the three key factors at the factory level. The advancement of their attributes and technologies provides opportunities of the realization of I4.0 [5]. Based on this comparison, which focuses mainly on the intelligence and consciousness aspects, a structural mode is given in Fig.1. It considers five levels of the implementation of CPS: connection, conversion, cyber, cognition and configuration [13]. 


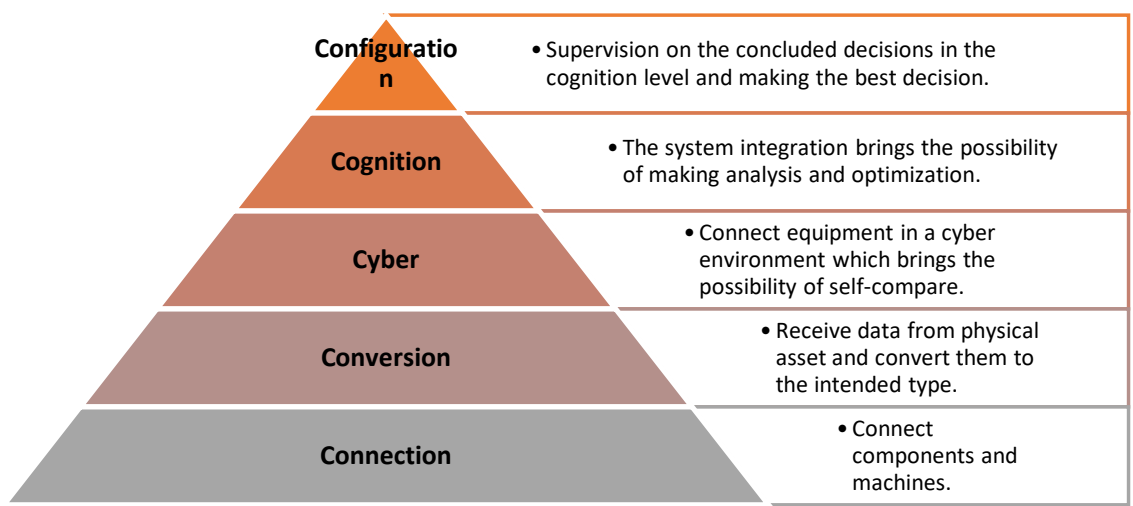

Fig. 1. 5C architecture of CPS implementation [13].

According to the 5C model, a comparison between I4.0 and current manufacturing systems where reconfigurable manufacturing is regarded as the most advanced method reveals that not only the maturity level of interoperability needs to be improved, but also the intelligence aspect is still a missing element. With the aim of addressing this shortage, a categorical framework in a matrix form consisting nine generic steps is provided, which considers three levels of intelligence (control, integration and intelligence) as well as three dimensions of automation (machine, process and factory) in order to achieve I4.0. This idea suggests that, within each dimension of automation, the improvement of intelligence level should be done in a systematic way following their sequence [10].

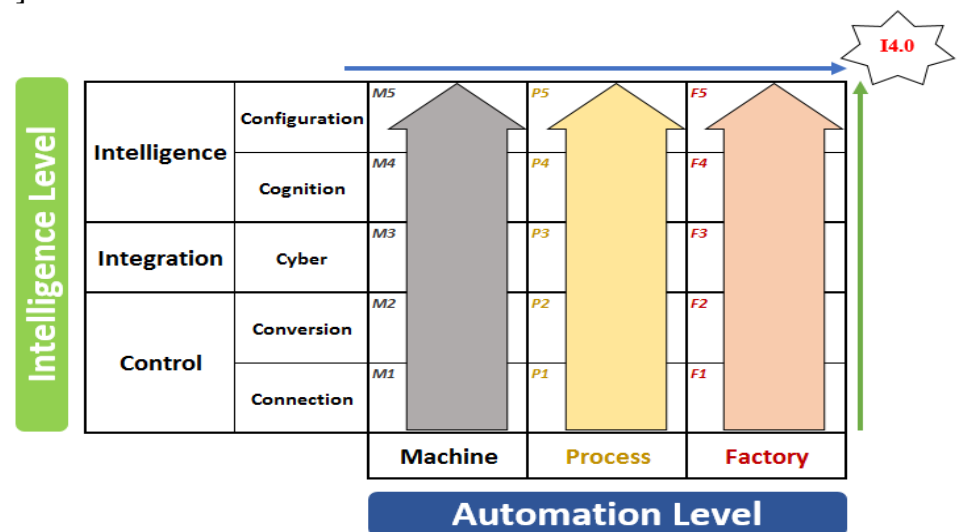

Fig. 2. A new approach of the categorical framework for CPS.

Intelligence is the common intellectual factor considered by both aforementioned ideas. In this paper, another approach has been derived, which categorizes the five levels of CPS within the three stages of intelligence and provides more elaboration of this aspect. This idea converts the categorical framework of CPS into a 15-step matrix with almost the same principles, as shown in Fig. 2. The main purpose of this model is to make a more perceptual roadmap of CPS and to provide a convergent framework for the realization of I4.0. 


\section{The Role of Virtual Technology}

The study of the nine technological components of I4.0 highlights simulation and Augmented Reality (AR) are two of the most important elements. Simulation provides a virtual, and mostly $3 \mathrm{D}$, environment to establish more opportunities for comparing different setups and optimizing the overall process. The general purpose of AR is to create an interface between factory and human or software [6]. A combination of both technologies leads to a more generic category: Virtual Technology (VT), which may potentially contribute to both aspects of CPS. With this perspective, VT provides a functional production system in a virtual environment where all the machines and components work together in an integrated and intelligent manner. Moreover, VT focuses on the interoperability aspect and opens up the possibility to test and implement new ideas, designs and algorithms.

The general attitude towards VT has been argued in many research works and been emerged in a variety of forms and terms within the scope of I4.0. One study regarding the intelligence aspect in the manufacturing sectors, mainly SMEs [9], demonstrates the significance of artificial intelligence for meeting the main features of an intelligent manufacturing system, i.e., flexibility and reconfigurability. This idea emphasizes the role of VTs, i.e., Virtual Reality (VR) and Virtual Manufacturing (VM), as the tools to improve the intelligence level and to achieve I4.0 [9]. Another novel paradigm is Virtual Factory (VF), which suggests to situate not only a part but also the entire factory in a software equipped with simulation module in order to incorporate all active elements in a factory. This idea emphasizes some features such as agility, scalability, and so forth. It considers four main elements for the implementation: reference model, virtual factory manager, functional modules and integration of knowledge [14]. Thanks to the possibility of providing a tangible virtual 3D environment, the widespread domain of VTs, i.e., VR, not only supports the simulation attribute but also discovers some critical issues of the new ideas before the implementation phase. In this regards, a research divides the dimensions of VR into three functional categories or phases: design, operation management and manufacturing processes [15]. This idea is one of the most important driving forces for manufacturers to give suggestions to software developers so that the focus of each simulation software can be specified.

Given the essence of VT in the field of I4.0, a simulation project is done in order to demonstrate a unified factory in a virtual environment by using Visual Components 4.1 simulation software (link: https://youtu.be/11Ax0OZUrEU). According to the aforementioned classification, this example focuses on the manufacturing process where all the equipment including machines, sensors, robots and other technical and generic facilities are integrated in a 3D graphical environment in order to form a fully automated factory. The main task of this factory is to produce four cylindrical parts considering quality control and reprocessing unit. After the packaging process, the products will be delivered to the customers at the intended batch size. Fig. 3 represents the sequential flowchart of the factory's logic.

The very first matter to be considered in a manufacturing process is the plant layout, which strikingly affects the internal logistics, i.e., flow of material. In this example, the layout is opted for U-Shape, as shown in Fig. 4(A). The integration and corporation 


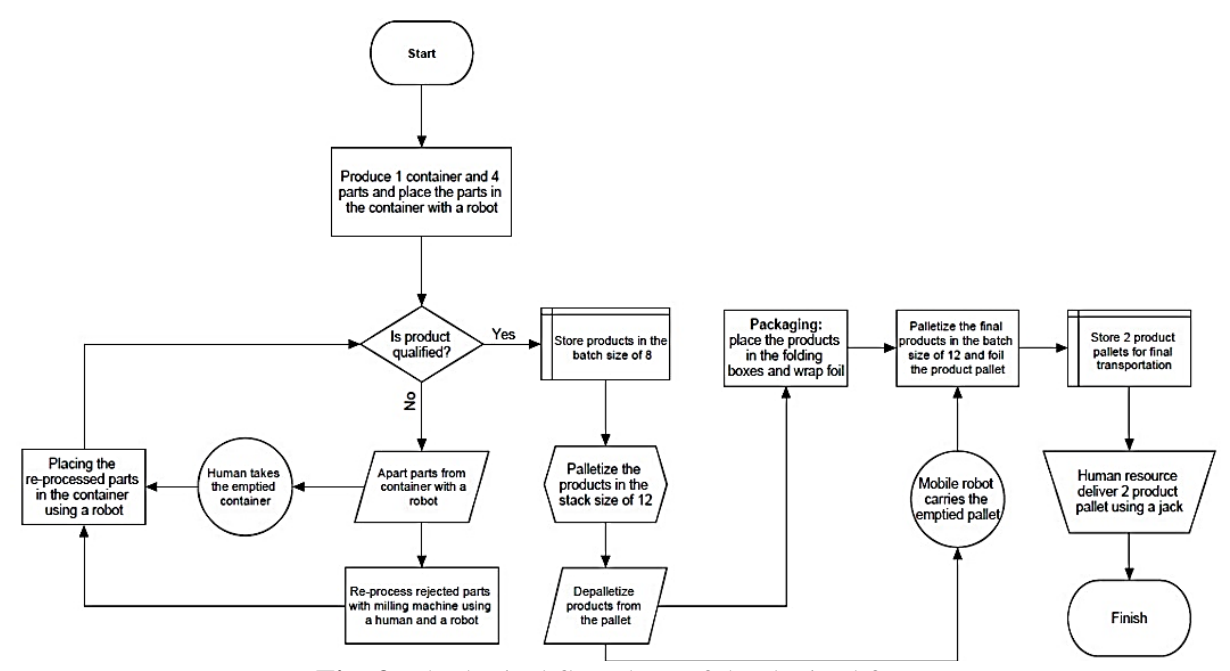

Fig. 3. The logical flowchart of the devised factory.

among modules and equipment is accomplished by python programming, which is not only used to control and unify facilities but also to provide the fundaments of increasing flexibility and agility. From a logistic point of view, this example provides some astonishing approaches. One example is the transportation of reprocessed parts, which is performed by using an AGV equipped with a universal robot, as shown in Fig. 4(B). This idea assists a company in decreasing some of the cost drivers, i.e., purchasing cost of the conveyors, maintenance expenses, etc. Moreover, this idea provides companies with opportunities to test different layout configurations.

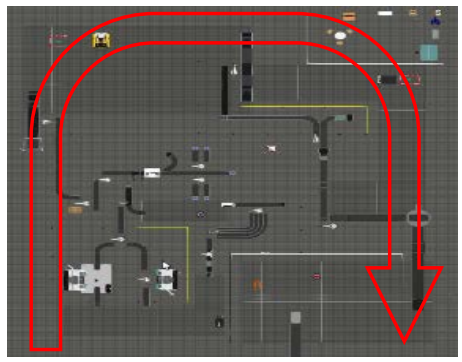

(A) Factory layout and flow of material

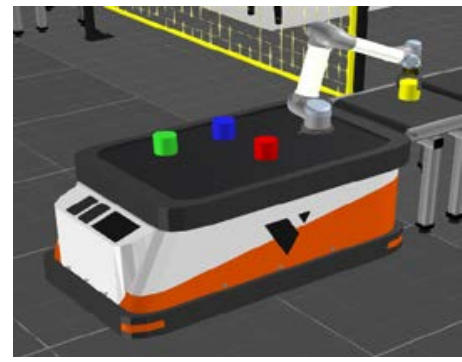

(B) AGV with a universal robot

Fig. 4. Simulation Environment.

Among many advantages of using a simulation software, this example contributes to outline the following ones:

- Modifying the plant layout and testing different designs in a quick and flexible manner.

- Altering the batch size and benchmarking different manufacturing strategies. 
- Making decision on whether to produce a specific product and evaluating the corresponding consequence.

\section{$4 \quad$ The Role of Digital Twin}

An ideal CPS connects all components and machines in order to provide a unified system in a cyber environment. This condition integrates the real factory with its virtual model in a simulation environment and provides a bi-directional connection and data flow between them in order to control the physical system while, at the same time, reflect the real change in the virtual environment [16]. The improvements on VTs are capable to provide such a unified system and enable the engineers to test and implement new ideas and intelligent algorithms. However, these advances are provided in a virtual environment, and the connection between the real world and the virtual environment is the most significant challenge. A new concept: Digital Twin (DT) aims to address this issue. It provides a digital representation of the physical components/machines of a real factory in a virtual environment where a real time and instant synchronization is established between them [17].

Inspired from the concept of DT, one research suggests improving the simulation softwares is an essential step in this regard [18]. It proposes a five-tier logic to establish a multi-layer simulation software based on the Model-View-View-Model (MVVM) paradigm and follows a sequence to simulate the DT of a simple process. Although it is only adaptable to a limited number of processes, the optimization feature can be considered as a noticeable achievement of this research [18]. Another approach proposes a concept: Versatile Simulation Database (VSD), which incorporates DT and Virtual Testbed in order to provide the possibility to achieve improved flexibility and functionality through integrating different types of simulation. Driving assistance systems, i.e., automatic parking system, can be referred to as a close instance in this regard [19]. Another industrial research presents a DT of a robotized press-brake processing line of a factory in South Korea with the use ROS Gazebo simulation, as shown in Fig. 5. In this process, the information related to the conveyors, parts, machines and robots is received by the sensors and robot controller, which is then reflected in the simulation environment. In order to establish the connection to control the physical elements, FlexGui 4.0 offers an online page to control the robots, machines and conveyors [20].

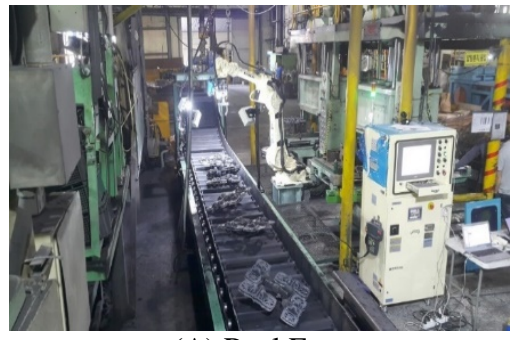

(A) Real Factory

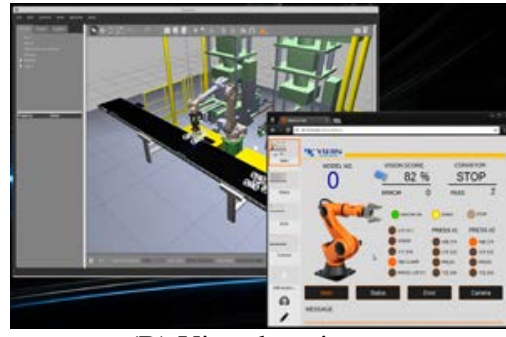

(B) Virtual environment

Fig. 5. DT of a robotized press-brake processing line. 
Based on the discussions above, DT is a promising technology in the realization of the concept of I4.0. Some advantages of DT are given as follows.

- $\quad$ Realizing a real-time synchronization between the physical elements and the corresponding virtual model.

- Bringing the possibility of controlling the physical factory through the simulation environment and monitoring the alterations of the physical system in the virtual model.

- Tracing the modifications of the product throughout the process by establishing the DT of the product as well as all equipment.

Despite all achievements of the concept of VTs, I4.0 is still in its infancy and much more development should be accomplished in order to realize this concept. In this regards, DT is essential to pave the way for this ambition and provides the possibility of bringing all novel ideas into the real world by satisfying the interoperability phase of CPS.

\section{Conclusion}

In this research, the role of VTs and DT in realizing the concept of I4.0 is thoroughly discussed. First, the concept of I4.0 is studied, where CPS and IoT are considered as the fundamental bases of it. Inspired from the recent development on the architecture of CPS, a categorical approach is proposed and elaborated to a higher degree in order to incorporate existing ideas and provide a unified and convergent framework for the realization of I4.0.

The study of the fundamental elements of I4.0 has revealed that VTs, i.e., Simulation and AR, etc., are among the most crucial technologies to realize the I4.0 concept, which provide an easy, flexible, visualized and cost effective way to evaluate and optimize different manufacturing scenarios, decisions, and process integration. However, establishing a bi-directional connection between the virtual environment and physical systems is still a significant challenge, which is currently under the focus of both academicians and practitioners.

This issue represents a huge gap between the current status of manufacturing system and the ideal condition in I4.0. In this regards, the concept of DT is introduced as the core module to address this challenge. DT provides a real-time and bi-directional connection, visualizes the product and equipment, and offers an integrated interface to control the physical system through the virtual environment. Consequently, DT not only enables the interoperability phase but also provides opportunities for improving the consciousness and intelligence.

\section{Acknowledgment}

This research is supported by TRINITY Project, which is financed under EU Horizon 2020 Programme. 


\section{References}

[1] J. Thangaraj and R. Lakshmi Narayanan, "Industry 1.0 to 4.0: The Evolution of Smart Factories," 2018.

[2] K. Schwab, The fourth industrial revolution, Currency, 2017.

[3] W. Krämer, Mittelstandsökonomik: Grundzüge einer umfassenden Analyse kleiner und mittlerer Unternehmen [SME Economics: Principles of a comprehensive analysis of SMEs], München, 2003.

[4] European Union, [Online]. Available: https://ec.europa.eu/growth/smes/business-friendly-environment/smedefinition_en.

[5] J. Lee, "Industry 4.0 in big data environment," German Harting Magazine, vol. 1, pp. 8-10, 2013.

[6] M. Rüßmann, M. Lorenz, P. Gerbert, M. Waldner, J. Justus, P. Engel and M. Harnisch, "Industry 4.0: The future of productivity and growth in manufacturing industries," Boston Consulting Group, vol. 9, pp. 54-89, 2015.

[7] G. Erboz, "How To Define Industry 4.0: Main Pillars Of Industry 4.0," in 7th International Conference on Management (ICoM 2017), At Nitra, Slovakia, 2017.

[8] H. Rahman and R. Rahmani, "Enabling distributed intelligence assisted Future Internet of Things Controller (FITC)," Applied computing and informatics, vol. 14, no. 1, pp. 73-87, 2018.

[9] T. Huang, W. D. Solvang and H. Yu, "An introduction of small-scale intelligent manufacturing system," in 2016 International Symposium on Small-scale Intelligent Manufacturing Systems (SIMS), Narvik, Norway, 2016.

[10] J. Qin, Y. Liu and R. Grosvenor, "A categorical framework of manufacturing for industry 4.0 and beyond," Procedia Cirp, vol. 52, pp. 173178, 2016.

[11] R. Baheti and H. Gill, "Cyber-physical systems," The impact of control technology, vol. 12, no. 1, pp. 161-166, 2011.

[12] E. Molina and E. Jacob, "Software-defined networking in cyber-physical systems: A survey," Computers \& Electrical Engineering, vol. 66, pp. 407419, 2018.

[13] J. Lee, B. Bagheri and H.-A. Kao, "A cyber-physical systems architecture for industry 4.0-based manufacturing systems," Manufacturing letters, vol. 3, pp. 18-23, 2015.

[14] M. Sacco, P. Pedrazzoli and W. Terkaj, "VFF: Virtual Factory Framework," in 2010 IEEE International Technology Management Conference (ICE), 2010. 
[15] T. S. Mujber, T. Szecsi and M. S. Hashmi, "Virtual reality applications in manufacturing process simulation," Journal of materials processing technology, vol. 155, pp. 1834-1838, 2004.

[16] J. Lee, "Smart factory systems," Informatik-Spektrum, vol. 38, no. 3, pp. 230-235, 2015.

[17] E. Negri, L. Fumagalli and M. Macchi, "A review of the roles of digital twin in cps-based production systems," Procedia Manufacturing, vol. 11, pp. 939-948, 2017.

[18] P. Tavares, J. A. Silva, P. Costa, G. Veiga and A. P. Moreira, "Flexible work cell simulator using digital twin methodology for highly complex systems in industry 4.0," in Iberian Robotics conference, 2017.

[19] M. Schluse and J. Rossmann, "From simulation to experimentable digital twins: Simulation-based development and operation of complex technical systems," in 2016 IEEE International Symposium on Systems Engineering (ISSE), 2016.

[20] B. Shu, G. Sziebig and B. Solvang, "Introduction of Cyber-Physical System in Robotized Press-Brake Line for Metal Industry," in International Workshop of Advanced Manufacturing and Automation, 2017. 